\title{
RENORMALIZATIONS OF MEASURABLE OPERATOR IDEAL SPACES AFFILIATED TO SEMI-FINITE VON NEUMANN ALGEBRA
}

\author{
A.M. BIKCHENTAEV
}

\begin{abstract}
This work is devoted to non-commutative analogues of classical methods of constructing functional spaces. Let a von Neumann algebra $\mathcal{M}$ of operators act in a Hilbert space $\mathcal{H}, \tau$ be a faithful normal semi-finite trace $\mathcal{M}$. Let $\widetilde{\mathcal{M}}$ be an $*$-algebra of $\tau$-measurable operators, $|X|=\sqrt{X^{*} X}$ for $X \in \widetilde{\mathcal{M}}$. A lineal $\mathcal{E}$ in $\widetilde{\mathcal{M}}$ is called ideal space on $(\mathcal{M}, \tau)$ if 1) $X \in \mathcal{E}$ implies $X^{*} \in \mathcal{E}$

2) $X \in \mathcal{E}, Y \in \widetilde{\mathcal{M}}$ and $|Y| \leqslant|X|$ imply $Y \in \mathcal{E}$.

Let $\mathcal{E}, \mathcal{F}$ be ideal spaces on $(\mathcal{M}, \tau)$. We propose a method of constructing a mapping $\tilde{\rho}: \mathcal{E} \rightarrow[0,+\infty]$ with nice properties by employing a mapping $\rho$ on a positive cone $\mathcal{E}^{+}$. At that, if $\mathcal{E}=\mathcal{M}$ and $\rho=\tau$, then $\tilde{\rho}(X)=\tau(|X|)$ and if the trace $\tau$ is finite, then $\tilde{\rho}(X)=\|X\|_{1}$ for all $X \in \mathcal{M}$. We study the case as $\tilde{\rho}(X)$ is equivalent to the original mapping $\rho(|X|)$. Employing mappings on $\mathcal{E}$ and $\mathcal{F}$, we construct a new mapping with nice properties on the sum $\mathcal{E}+\mathcal{F}$. We provide examples of such mappings. The results are new also for $*$-algebra $\mathcal{M}=\mathcal{B}(\mathcal{H})$ of all bounded linear operators in $\mathcal{H}$ equipped with a canonical trace $\tau=\operatorname{tr}$.
\end{abstract}

Keywords: Hilbert space, linear operator, von Neumann algebra, normal trace, measurable operators, ideal space, renormalization.

Mathematics Subject Classification: 46L10; 47C15; 46L51

\section{INTRODUCTION}

The work is devoted to non-commutative analogues of classical methods of constructing functional spaces. The beginning of developing a corresponding aspect of the theory of noncommutative integration is due to I.E. Sigal and J. Dixmier, who in the beginning of 1950s created the theory of non-commutative integration with respect to the trace on a semi-finite von Neumann algebra [1]. The results of their studies were effectively applied in the duality theory for unimodular groups and stimulated a progress in "non-commutative probability theory". The theory of algebras of measurable and locally measurable operator is being intensively developed and has interesting applications in various fields of functional analysis, mathematical physics, statistical mechanics, quantum field theory.

Till the mid of 1980s, the ideal spaces of measurable operators served mostly as an object of studying, see [2] and the references therein. Recently there appeared publications in which they served as a tool, see, for instance, [3]. This demonstrates a topicality of finding new methods for constructing new methods for constructing ideal spaces and developing a general theory of

A.M. Bikchentaev, Renormalizations of measurable operator ideal spaces afFiliated to semiFINITE VON NEUMANN ALGEBRA.

(c) Bikchentaev A.M. 2019.

The work is supported by subsidy granted to Kazan Federal University for making a state task in the field of scientific activity (1.9773.2017/8.9).

Submitted August 22, 2018. 
these spaces. In [4], [5] new methods were proposed for constructing ideal spaces on semi-finite von Neumann algebras and the geometric and topological spaces of the obtained spaces were studied.

Let a von Neumann algebra $\mathcal{M}$ of operators act in a Hilbert space $\mathcal{H}, \tau$ be a faithful normal semi-infinite trace on $\mathcal{M}$. Let $\mathcal{E}, \mathcal{F}$ be ideal spaces on $(\mathcal{M}, \tau)$. In Section 3 we propose a method of constructing a mapping $\tilde{\rho}: \mathcal{E} \rightarrow[0,+\infty]$ with nice properties by means of a given mapping $\rho$ on a positive cone $\mathcal{E}^{+}$. At that, if $\mathcal{E}=\mathcal{M}$ and $\rho=\tau$, then $\tilde{\rho}(X)=\tau(|X|)$ and if the trace $\tau$ is finite, then $\tilde{\rho}(X)=\|X\|_{1}$ for each $X \in \mathcal{M}$. We also study the case when $\tilde{\rho}(X)$ is equivalent to the initial mapping $\rho(|X|)$. Employing mappings onto $\mathcal{E}$ and $\mathcal{F}$, in Section 4 we construct a new mapping with nice properties on the sum $\mathcal{E}+\mathcal{F}$. The results are new also for $*$-algebra $\mathcal{M}=\mathcal{B}(\mathcal{H})$ of all bounded linear operators in $\mathcal{H}$ equipped by a canonical trace $\tau=\operatorname{tr}$.

\section{NotATIONS AND DEFINITIONS}

Let $\mathcal{M}$ be a von Neumann algebra in a Hilbert space $\mathcal{H}, \mathcal{M}^{\text {pr }}$ be a lattice of projectors $\left(P=P^{2}=P^{*}\right)$ in $\mathcal{M}, I$ be the identity mapping $\mathcal{M}, P^{\perp}=I-P$ for $P \in \mathcal{M}^{\text {pr }}, \mathcal{M}^{+}$be a cone of positive elements in $\mathcal{M}$, and $\mathcal{M}_{1}=\{X \in \mathcal{M}:\|X\| \leqslant 1\}$.

The mapping $\varphi: \mathcal{M}^{+} \rightarrow[0,+\infty]$ is called trace if $\varphi(X+Y)=\varphi(X)+\varphi(Y), \varphi(\lambda X)=\lambda \varphi(X)$ for each $X, Y \in \mathcal{M}^{+}, \lambda \geqslant 0$ (at that $0 \cdot(+\infty) \equiv 0$ ) and $\varphi\left(Z^{*} Z\right)=\varphi\left(Z Z^{*}\right)$ for each $Z \in \mathcal{M}$. A trace $\varphi$ is called faithful if $\varphi(X)>0$ for each $X \in \mathcal{M}^{+}, X \neq 0$; and it is semi-infinite if

$$
\varphi(X)=\sup \left\{\varphi(Y): Y \in \mathcal{M}^{+}, Y \leqslant X, \varphi(Y)<+\infty\right\}
$$

for each $X \in \mathcal{M}^{+}$. A trace is normal if

$$
X_{i} \nearrow X \quad\left(X_{i}, X \in \mathcal{M}^{+}\right) \Rightarrow \varphi(X)=\sup \varphi\left(X_{i}\right)
$$

see [6, Ch. V, Sect. 2].

An operator in $\mathcal{H}$ (not necessarily bounded of densely defined) is called affiliated to a von Neumann algebra $\mathcal{M}$ if it commutes with each unitary operator in the commutator subgroup $\mathcal{M}^{\prime}$ of the algebra $\mathcal{M}$. Hereafter $\tau$ is a faithful normal semi-infinite trace on $\mathcal{M}$. A closed operator $X$ affiliated to $\mathcal{M}$ with a domain $\mathcal{D}(X)$ dense in $\mathcal{H}$ is called $\tau$-measurable if for each $\varepsilon>0$ there exists $P \in \mathcal{M}^{\text {pr }}$, such that $P \mathcal{H} \subset \mathcal{D}(X)$ and $\tau\left(P^{\perp}\right)<\varepsilon$. A set $\widetilde{\mathcal{M}}$ of all $\tau$-measurable operators is an $*$-algebra with respect to the operations of taking the adjoint operator, multiplication by a scalar and the operators of strong addition and multiplication obtained by closure of usual operators [1], [7]. For a family $\mathcal{L} \subset \widetilde{\mathcal{M}}$ by $\mathcal{L}^{+}$and $\mathcal{L}^{\text {sa }}$ we denote its positive and Hermitian part, respectively. A partial order in $\widetilde{\mathcal{M}}^{\text {sa }}$ generated by a proper cone $\widetilde{\mathcal{M}}^{+}$is denoted by $\leqslant$. If $X \in \widetilde{\mathcal{M}}$ and $X=U|X|$ is the polar decomposition of $X$, then $U \in \mathcal{M}_{1}$ and $|X|=\sqrt{X^{*} X} \in \widetilde{\mathcal{M}}^{+}$.

The $*$-algebra $\widetilde{\mathcal{M}}$ is equipped with the topology $t_{\tau}$ of convergence measure [7], whose fundamental system of neighbourhood is formed by the sets

$$
U(\varepsilon, \delta)=\left\{X \in \widetilde{\mathcal{M}}: \exists P \in \mathcal{M}^{\mathrm{pr}}\left(\|X P\| \leqslant \varepsilon \text { and } \tau\left(P^{\perp}\right) \leqslant \delta\right)\right\}, \quad \varepsilon>0, \quad \delta>0 .
$$

It is know that $\left(\widetilde{\mathcal{M}}, t_{\tau}\right)$ is a complete metrizable topological *-algebra and $\mathcal{M}$ is dense in $\left(\widetilde{\mathcal{M}}, t_{\tau}\right)$.

By $\mu_{t}(X)$ we denote a permutation of an operator $X \in \widetilde{\mathcal{M}}$, that is, a non-decreasing rightcontinuous function $\mu(X):(0, \infty) \rightarrow[0, \infty)$ defined by the formula

$$
\mu_{t}(X)=\inf \left\{\|X P\|: \quad P \in \mathcal{M}^{\mathrm{pr}}, \tau\left(P^{\perp}\right) \leqslant t\right\}, \quad t>0 .
$$


The set of $\tau$-compact operators $\widetilde{\mathcal{M}}_{0}=\left\{X \in \widetilde{\mathcal{M}}: \lim _{t \rightarrow \infty} \mu_{t}(X)=0\right\}$ is a $t_{\tau}$-closed ideal in $\widetilde{\mathcal{M}}$ [8]. Let $m$ be a Lebesgue linear measure on $\mathbb{R}$. An associated with $(\mathcal{M}, \tau)$ non-commutative Lebesgue $L_{p}$-space $(0<p<\infty)$ can be defined as $L_{p}(\mathcal{M}, \tau)=\left\{X \in \widetilde{\mathcal{M}}: \mu_{t}(X) \in L_{p}\left(\mathbb{R}^{+}, m\right)\right\}$ with $F$-norm (norm for $1 \leqslant p<\infty)\|X\|_{p}=\left\|\mu_{t}(X)\right\|_{p}, X \in L_{p}(\mathcal{M}, \tau)$.

A lineal $\mathcal{E}$ in $\widetilde{\mathcal{M}}$ is called ideal space on $(\mathcal{M}, \tau)$, see [9], [3], 2], if

1) $X \in \mathcal{E}$ implies $X^{*} \in \mathcal{E}$

2) $X \in \mathcal{E}, Y \in \widetilde{\mathcal{M}}$ and $|Y| \leqslant|X|$ imply $Y \in \mathcal{E}$.

This is, for instance, the algebra $\mathcal{M}$, the set of elementary operators $\mathcal{F}(\mathcal{M}), \widetilde{\mathcal{M}}_{0},\left(L_{1}+\right.$ $\left.L_{\infty}\right)(\mathcal{M}, \tau)$ and $L_{p}(\mathcal{M}, \tau)$ as $0<p<+\infty$. For each ideal space $\mathcal{E}$ on $(\mathcal{M}, \tau)$ we have $\mathcal{M E} \mathcal{M} \subseteq \mathcal{E}$ [2, Lm. 5].

If $\mathcal{M}=\mathcal{B}(\mathcal{H})$ is $*$-algebra of all bounded linear operators in $\mathcal{H}$ and $\tau=\operatorname{tr}$ is the canonical trace, then $\widetilde{\mathcal{M}}$ and $\widetilde{\mathcal{M}}_{0}$ coincide with $\mathcal{B}(\mathcal{H})$ and the ideal of compact operators in $\mathcal{H}$, respectively. We have

$$
\mu_{t}(X)=\sum_{n=1}^{\infty} s_{n}(X) \chi_{[n-1, n)}(t), \quad t>0,
$$

where $\left\{s_{n}(X)\right\}_{n=1}^{\infty}$ is the sequence of $s$-numbers of a compact operator $X[10] ; \chi_{A}$ is the indicator of a set $A \subset \mathbb{R}$. Then the space $L_{p}(\mathcal{M}, \tau)$ is the Schatten-von Neumann ideal $\mathfrak{S}_{p}, 0<p<\infty$.

Lemma 2.1. [11]. If $X, Y \in \widetilde{\mathcal{M}}^{+}$and $X \leqslant Y$, then there exists an operator $Z \in \mathcal{M}_{1}$ such that $\sqrt{X}=Z \sqrt{Y}$ and $X=Z Y Z^{*}$.

Lemma 2.2. [12] If $X, Y \in \widetilde{\mathcal{M}}^{\text {sa }}$ and $Z \in \widetilde{\mathcal{M}}$, then the inequality $X \leqslant Y$ implies $Z X Z^{*} \leqslant$ $Z Y Z^{*}$.

\section{RENORMALizATION OF IDEAL SPACES}

Let $\tau$ be a faithful normal semi-infinite trace on a von Neumann algebra $\mathcal{M}, \mathcal{E}$ be an ideal space on $(\mathcal{M}, \tau)$. If $A \in \widetilde{\mathcal{M}}$ and $A^{*} A \in \mathcal{E}$, then $A A^{*} \in \mathcal{E}$ [2, Lm. 5]. We assume the following conditions for a mapping $\rho: \mathcal{E}^{+} \rightarrow[0,+\infty]$ :

(i) if $X, Y \in \mathcal{E}^{+}$and $X \leqslant Y$, then $\rho(X) \leqslant \rho(Y)$;

(ii) $\rho\left(X^{*} X\right)=\rho\left(X X^{*}\right)$ for each $X \in \widetilde{\mathcal{M}}$ with $X^{*} X \in \mathcal{E}$;

(iii) $\rho(X+Y) \leqslant \rho(X)+\rho(Y)$ for each $X, Y \in \mathcal{E}^{+}$.

We assume the following conditions for a mapping $\rho: \mathcal{E} \rightarrow[0,+\infty]$ :

(iv) $\rho(X)=\rho(|X|)=\rho\left(X^{*}\right)$ for each $X \in \mathcal{E}$;

(v) $\rho(X+Y) \leqslant \rho(X)+\rho(Y)$ for each $X, Y \in \mathcal{E}$;

(vi) $\rho(\lambda X)=|\lambda| \rho(X)$ for each $\lambda \in \mathbb{C}$ and $X, Y \in \mathcal{E}$ (at that, $0 \cdot+\infty=0$ ).

Example 3.1. Let $\left(\mathcal{E},\|\cdot\|_{\mathcal{E}}\right)$ be a normalized ideal space on $(\mathcal{M}, \tau)[9]$. Then the restriction of the norm $\|\cdot\|_{\mathcal{E}}$ on $\mathcal{E}^{+}$satisfies conditions (i)-(iii), while $\|\cdot\|_{\mathcal{E}}$ satisfies conditions (iv)-(vi). The examples are $(\mathcal{M},\|\cdot\|)$ and $\left(L_{p}(\mathcal{M}, \tau),\|\cdot\|_{p}\right)$ for $p \geqslant 1$.

Example 3.2. Let $\rho=\langle(\cdot) \xi, \xi\rangle(\xi \in \mathcal{H},\|\xi\|=1)$ be a vector state on the algebra $\mathcal{B}(\mathcal{H})$. The restriction $\left.\rho\right|_{\mathcal{B}(\mathcal{H})}+$ satisfies conditions (i) and (iii). For $\rho_{1}=|\rho|$, conditions (v) and (vi) are satisfied.

Example 3.3. Let $\mathcal{E}$ be an ideal space on $(\mathcal{M}, \tau)$ and $Y \in \mathcal{E}^{+}$. We let

$$
\rho(X)=\inf \{\lambda>0: X \leqslant \lambda Y\}
$$

for each $X \in \mathcal{E}^{+}$assuming that inf over $\varnothing$ is equal to $+\infty$. Then $\rho$ satisfies conditions (i) and (iii). 
Proposition 3.1. Let $\mathcal{E}$ be an ideal space on $(\mathcal{M}, \tau)$ and a mapping $\rho: \mathcal{E} \rightarrow[0,+\infty]$ satisfy conditions (iv)-(vi) (or (i), (iv) and (v)). Then $\mathcal{F}=\{X \in \mathcal{E}: \rho(X)<+\infty\}$ is an ideal space on $(\mathcal{M}, \tau)$.

Proposition 3.2. Let $\mathcal{E}$ be an ideal space on $(\mathcal{M}, \tau)$ and a mapping $\rho: \mathcal{E} \rightarrow[0,+\infty]$ satisfy conditions (i)-(iii). Then

$$
\rho(X) \leqslant \sum_{k=1}^{n} \rho\left(Y_{k} X Y_{k}^{*}\right)
$$

for each $X \in \mathcal{E}^{+}$and $\left\{Y_{k}\right\}_{k=1}^{n} \subset \mathcal{M}$ with $\sum_{k=1}^{n} Y_{k}^{*} Y_{k} \geqslant I$.

Proof. By Lemma 2.2 we have

$$
X \leqslant \sqrt{X}\left(\sum_{k=1}^{n} Y_{k}^{*} Y_{k}\right) \sqrt{X}=\sum_{k=1}^{n} \sqrt{X} Y_{k}^{*} Y_{k} \sqrt{X}
$$

This is why

$$
\rho(X) \leqslant \sum_{k=1}^{n} \rho\left(\sqrt{X} Y_{k}^{*} Y_{k} \sqrt{X}\right)=\sum_{k=1}^{n} \rho\left(Y_{k} X Y_{k}^{*}\right)
$$

and this completes the proof.

Lemma 3.1. Let $\mathcal{E}$ be an ideal space on $(\mathcal{M}, \tau)$ and a mapping $\rho: \mathcal{E} \rightarrow[0,+\infty]$ satisfies conditions (i), (iv). If $X, Y \in \mathcal{M}_{1}$, then $\rho(X Z Y) \leqslant \rho(Z)$ for each $Z \in \mathcal{E}$.

Proof. If for some operators we have $A, B \in \mathcal{M}$, then $A Z B \in \mathcal{E}$ for each $Z \in \mathcal{E}$. For each $X \in \mathcal{M}_{1}$ and $Z \in \mathcal{E}$, by the operator monotonicity of the function $\lambda \mapsto \sqrt{\lambda}$ on $\mathbb{R}^{+}$and Lemma 2.2 we have

$$
\rho(X Z)=\rho(|X Z|)=\rho\left(\sqrt{Z^{*} X^{*} X Z}\right) \leqslant \rho\left(\sqrt{Z^{*} Z}\right)=\rho(|Z|)=\rho(Z) .
$$

If $Y \in \mathcal{M}_{1}$, then $Y^{*} \in \mathcal{M}_{1}$ and $\rho(Z Y)=\rho\left((Z Y)^{*}\right)=\rho\left(Y^{*} Z^{*}\right) \leqslant \rho\left(Z^{*}\right)=\rho(Z)$. The proof is complete.

Proposition 3.3. Let $\mathcal{E}$ be an ideal space on $(\mathcal{M}, \tau)$ and a mapping $\rho: \mathcal{E} \rightarrow[0,+\infty]$ satisfies conditions (i), (iv). Then $\rho$ satisfies condition (ii).

Proof. Let $X=U|X|$ be the polar decomposition of the operator $X \in \widetilde{\mathcal{M}}$. Then $U, U^{*} \in \mathcal{M}_{1}$ and $X X^{*}=U X^{*} X U^{*} \in \mathcal{E}$. By Lemma 3.1 we have

$$
\rho\left(X X^{*}\right)=\rho\left(U X^{*} X U^{*}\right) \leqslant \rho\left(X^{*} X\right) .
$$

Replacing $X$ by $X^{*}$, we obtain $\rho\left(X^{*} X\right) \leqslant \rho\left(X X^{*}\right)$ and this proves (ii).

Theorem 3.1. Let $\mathcal{E}$ be an ideal space on $(\mathcal{M}, \tau)$ and a mapping $\rho: \mathcal{E}^{+} \rightarrow[0,+\infty]$ be given. We denote

$$
\tilde{\rho}(X)=\sup _{Z \in \mathcal{M}_{1}} \sup _{0 \leqslant A \leqslant Z|X| Z^{*}} \rho(A) \quad \text { for each } \quad X \in \mathcal{E} .
$$

Then $\tilde{\rho}$ satisfies conditions (i), (ii), (iv) and $\tilde{\rho}(X) \geqslant \rho(|X|)$ for each $X \in \mathcal{E}$.

Proof. Let $X, Y \in \mathcal{E}^{+}$and $X \leqslant Y$. By Lemma 2.1, there exists an operator $U \in \mathcal{M}_{1}$ such that $X=U Y U^{*}$. This is why

$$
\tilde{\rho}(X)=\sup _{Z \in \mathcal{M}_{1}} \sup _{0 \leqslant A \leqslant Z X Z^{*}} \rho(A)=\sup _{Z \in \mathcal{M}_{1}} \sup _{0 \leqslant A \leqslant Z U Y U^{*} Z^{*}} \rho(A) \leqslant \sup _{Z \in \mathcal{M}_{1}} \sup _{0 \leqslant A \leqslant Z Y Z^{*}} \rho(A)=\tilde{\rho}(Y)
$$

and this proves (i). 
Let $X=U|X|$ be the polar decomposition of an operator $X \in \widetilde{\mathcal{M}}$. Then $\left|X^{*}\right|=U|X| U^{*}$ and $\left|X^{*}\right|^{2}=U|X|^{2} U^{*}$. We have

$$
\tilde{\rho}\left(X^{*}\right)=\sup _{Z \in \mathcal{M}_{1}} \sup _{0 \leqslant A \leqslant Z\left|X^{*}\right| Z^{*}} \rho(A)=\sup _{Z \in \mathcal{M}_{1}} \sup _{0 \leqslant A \leqslant Z U|X| U^{*} Z^{*}} \rho(A) \leqslant \sup _{Z \in \mathcal{M}_{1}} \sup _{0 \leqslant A \leqslant Z|X| Z^{*}} \rho(A)=\tilde{\rho}(X) .
$$

Thanks to the identity $\left(X^{*}\right)^{*}=X$, this proves (iv). Now (ii) follows Proposition 3.3. The proof is complete.

Theorem 3.2. Let $\mathcal{E}$ be an ideal space on $(\mathcal{M}, \tau)$ and a mapping $\rho: \mathcal{E}^{+} \rightarrow[0,+\infty]$ satisfy condition (iii). Then the mapping $\tilde{\rho}: \mathcal{E} \rightarrow[0,+\infty]$ defined by formula (1) satisfies condition (v).

Proof. Let $X, Y \in \mathcal{E}$ and $\alpha=\tilde{\rho}(X+Y)$. Then for each number $\varepsilon>0$ there exist operators $Z_{\varepsilon} \in \mathcal{M}_{1}$ and $A_{\varepsilon} \in \mathcal{E}^{+}$such that

$$
A_{\varepsilon} \leqslant Z_{\varepsilon}|X+Y| Z_{\varepsilon}^{*}, \quad \alpha \geqslant \rho\left(A_{\varepsilon}\right)>\alpha-\varepsilon .
$$

There exist partial isometries $V, W \in \mathcal{M}_{1}$ such that $|X+Y| \leqslant V|X| V^{*}+W|Y| W^{*}$, see [13, Thm. 2.2], [14]. This is why, cf. also Lemma 2.2, for each number $\varepsilon>0$ there exist operators $Z_{\varepsilon} \in \mathcal{M}_{1}$ and $A_{\varepsilon} \in \mathcal{E}^{+}$such that

$$
A_{\varepsilon} \leqslant Z_{\varepsilon} V|X| V^{*} Z_{\varepsilon}^{*}+Z_{\varepsilon} W|Y| W^{*} Z_{\varepsilon}^{*}, \quad \alpha \geqslant \rho\left(A_{\varepsilon}\right)>\alpha-\varepsilon .
$$

By Lemma 2.1, for each number $\varepsilon>0$ there exists an operator $U_{\varepsilon} \in \mathcal{M}_{1}$ such that

$$
A_{\varepsilon}=U_{\varepsilon} Z_{\varepsilon} V|X| V^{*} Z_{\varepsilon}^{*} U_{\varepsilon}^{*}+U_{\varepsilon} Z_{\varepsilon} W|Y| W^{*} Z_{\varepsilon}^{*} U_{\varepsilon}^{*} .
$$

The operators $U_{\varepsilon} Z_{\varepsilon} V, U_{\varepsilon} Z_{\varepsilon} W$ belong to $\mathcal{M}_{1}$ and

$$
\rho\left(A_{\varepsilon}\right) \leqslant \rho\left(U_{\varepsilon} Z_{\varepsilon} V|X| V^{*} Z_{\varepsilon}^{*} U_{\varepsilon}^{*}\right)+\rho\left(U_{\varepsilon} Z_{\varepsilon} W|Y| W^{*} Z_{\varepsilon}^{*} U_{\varepsilon}^{*}\right) \leqslant \tilde{\rho}(X)+\tilde{\rho}(Y) .
$$

By the arbitrary choice of the number $\varepsilon>0$ we obtain $\alpha \leqslant \tilde{\rho}(X)+\tilde{\rho}(Y)$ and this completes the proof.

Remark 3.1. Under the assumptions of Theorem 3.1, if $\rho(A)=0 \Leftrightarrow A=0\left(A \in \mathcal{E}^{+}\right)$, then $\tilde{\rho}(X)=0 \Leftrightarrow X=0(X \in \mathcal{E})$; if $\rho(\lambda A)=\lambda \rho(A)$ for each $\lambda \in \mathbb{R}^{+}$and $A \in \mathcal{E}^{+}$, then $\tilde{\rho}$ satisfies condition (iv). If $\rho$ satisfies condition (i), then $\tilde{\rho}(I)=\rho(I)$ and

$$
\tilde{\rho}(X)=\sup _{Z \in \mathcal{M}_{1}} \rho\left(Z|X| Z^{*}\right) \quad \text { for each } \quad X \in \mathcal{E} .
$$

Proposition 3.4. Let $\mathcal{E}$ be an ideal space on $(\mathcal{M}, \tau), \rho: \mathcal{E}^{+} \rightarrow[0,+\infty]$, and a mapping $\tilde{\rho}: \mathcal{E} \rightarrow[0,+\infty]$ is defined by formula (1). If $\rho$ satisfies conditions (i) and (ii), then $\tilde{\rho}(X)=$ $\rho(|X|)$ for each $X \in \mathcal{E}$.

Proof. For each $Z \in \mathcal{M}_{1}$ we have $Z^{*} Z \leqslant I$ and $\sqrt{|X|} Z^{*} Z \sqrt{|X|} \leqslant|X|$ holds for each $X \in \mathcal{E}$ by Lemma 2.2. Then

$$
\tilde{\rho}(X)=\sup _{Z \in \mathcal{M}_{1}} \sup _{0 \leqslant A \leqslant Z|X| Z^{*}} \rho(A)=\sup _{Z \in \mathcal{M}_{1}} \rho\left(Z|X| Z^{*}\right)=\sup _{Z \in \mathcal{M}_{1}} \rho\left(\sqrt{|X|} Z^{*} Z \sqrt{|X|}\right)=\rho(|X|) .
$$

Thus, the restriction $\left.\tilde{\rho}\right|_{\mathcal{E}^{+}}$coincides with $\rho$. The proof is complete.

Example 3.4. Let $\mathcal{E}=\mathcal{M}$ and $\rho=\tau$. Then $\tilde{\rho}(X)=\tau(|X|)$ and if the trace $\tau$ is finite, then $\tilde{\rho}(X)=\|X\|_{1}$ for each $X \in \mathcal{M}$.

Proposition 3.5. Let $\mathcal{E}$ be an ideal space on $(\mathcal{M}, \tau), \rho: \mathcal{E}^{+} \rightarrow[0,+\infty]$, and let a mapping $\tilde{\rho}: \mathcal{E} \rightarrow[0,+\infty]$ is defined by formula (1). If the following conditions hold:

(vii) there exists $C_{1}>0$ such that $\rho\left(X^{*} X\right) \leqslant C_{1} \rho\left(X X^{*}\right)$ for each $X \in \widetilde{\mathcal{M}}$ with $X^{*} X \in \mathcal{E}$;

(viii) there exists $C_{2}>0$ such that $\rho(X) \leqslant C_{2} \rho(Y)$ for each $X, Y \in \mathcal{E}^{+}$with $X \leqslant Y$, then $\rho(|X|) \leqslant \tilde{\rho}(X) \leqslant 2 C_{1} C_{2}^{2} \rho(|X|)$ for each $X \in \mathcal{E}$. 
Proof. Let $X \in \mathcal{E}, Z \in \mathcal{M}_{1}$ and $0 \leqslant A \leqslant Z|X| Z^{*}$ be such that $\tilde{\rho}(X) \leqslant 2 \rho(A)$. By Lemma 2.2 we have $\sqrt{|X|} Z^{*} Z \sqrt{|X|} \leqslant|X|$ and

$$
\tilde{\rho}(X) \leqslant 2 \rho(A) \leqslant 2 C_{2} \rho\left(Z|X| Z^{*}\right) \leqslant 2 C_{1} C_{2} \rho\left(\sqrt{|X|} Z^{*} Z \sqrt{|X|}\right) \leqslant 2 C_{1} C_{2}^{2} \rho(|X|) .
$$

Thus, $\rho(|X|) \leqslant \tilde{\rho}(X) \leqslant 2 C_{1} C_{2}^{2} \rho(|X|)$ for each $X \in \mathcal{E}$. The proof is complete.

Remark 3.2. Let $\mathcal{E}$ be an ideal space on $(\mathcal{M}, \tau)$, where $\mathcal{M}$ is a finite von Neumann algebra, that is, $0 U \in \mathcal{M}$ and $U^{*} U=I$ imply $U U^{*}=I$. Assume that a mapping $\rho: \mathcal{E} \rightarrow \mathbb{R}^{+}$satisfies condition (v). We let

$$
\rho_{1}(X)=\sup _{Z, T \in \mathcal{M}_{1}} \rho(Z X T) \quad \text { for each } \quad X \in \mathcal{E} .
$$

It was shown in [15, Thm. 2] that a mapping $\rho_{1}: \mathcal{E} \rightarrow[0,+\infty]$ satisfies conditions (i), (iv) and (v). For a mapping $\rho$ with property (vi), the mapping $\rho_{1}$ also satisfies (vi).

Proposition 3.6. Let $\rho_{2}=\widetilde{\rho \mid \mathcal{E}^{+}}$. Then $\rho_{2}(X) \leqslant \rho_{1}(X)$ for each $X \in \mathcal{E}$.

Proof. Let $X \in \mathcal{E}$ and $\alpha=\rho_{2}(X)$. Then for each number $\varepsilon>0$ there exist operators $Z_{\varepsilon} \in \mathcal{M}_{1}$ and $A_{\varepsilon} \in \mathcal{E}^{+}$such that

$$
A_{\varepsilon} \leqslant Z_{\varepsilon}|X| Z_{\varepsilon}^{*}, \quad \alpha \geqslant \rho\left(A_{\varepsilon}\right)>\alpha-\varepsilon .
$$

By Lemma 2.1, for each number $\varepsilon>0$, there exists an operator $U_{\varepsilon} \in \mathcal{M}_{1}$ such that

$$
A_{\varepsilon}=U_{\varepsilon} Z_{\varepsilon}|X| Z_{\varepsilon}^{*} U_{\varepsilon}^{*}
$$

Hence,

$$
\rho_{1}(X)=\rho_{1}(|X|)=\sup _{Z, T \in \mathcal{M}_{1}} \rho(Z|X| T) \geqslant \rho\left(A_{\varepsilon}\right)>\alpha-\varepsilon .
$$

By an arbitrary choice of the number $\varepsilon>0$ we have $\rho_{1}(X) \geqslant \alpha$ and this completes the proof.

Example 3.5. Let a mapping $\rho: \mathcal{M}^{\mathrm{pr}} \rightarrow \mathbb{R}^{+}$is monotone and unitarily invariant. Then the mapping $\rho_{s}: \widetilde{\mathcal{M}} \rightarrow \mathbb{R}^{+}$defined by the formula $\rho_{s}(A)=\rho(s(|A|))$, where $A \in \widetilde{\mathcal{M}}$ and $s(|A|)$ is the support of the operator $|A|$ satisfies conditions (i), (ii) and (iv).

\section{NORMALizATION OF SUMS OF IDEAL SPACES}

If $\mathcal{E}, \mathcal{F}$ are ideal spaces on $(\mathcal{M}, \tau)$, then the sets $\mathcal{E} \cap \mathcal{F}$ and $\mathcal{E}+\mathcal{F}=\{A+B: A \in \mathcal{E}, B \in \mathcal{F}\}$ are also ideal spaces on $(\mathcal{M}, \tau)$ [2, Thm. 2]. The structure of ideal spaces is modular: if $\mathcal{E}, \mathcal{F}$ and $\mathcal{G}$ are ideal spaces on $(\mathcal{M}, \tau)$ and $\mathcal{E} \subset \mathcal{G}$, then $(\mathcal{E}+\mathcal{F}) \cap \mathcal{G}=\mathcal{E}+(\mathcal{F} \cap \mathcal{G})$ [2, Thm. 3]. In the theory of non-commutative integration, the space $\left(L_{1}+L_{\infty}\right)(\mathcal{M}, \tau)=L_{1}(\mathcal{M}, \tau)+\mathcal{M}$ plays an important role [12].

Theorem 4.1. Let $\mathcal{E}, \mathcal{F}$ be ideal spaces on $(\mathcal{M}, \tau)$ and $\mathcal{G}=\mathcal{E}+\mathcal{F}$. If mappings $\rho_{1}: \mathcal{E} \rightarrow$ $[0,+\infty]$ and $\rho_{2}: \mathcal{F} \rightarrow[0,+\infty]$ satisfy conditions (i), (iv), then the mapping $\rho: \mathcal{G} \rightarrow[0,+\infty]$ defined by the formula

$$
\rho(Z)=\inf \left\{\rho_{1}(X)+\rho_{2}(Y): X \in \mathcal{E}, Y \in \mathcal{F} \text { and } Z=X+Y\right\},
$$

also satisfies conditions (i), (iv).

Proof. Let $Z=U|Z|$ be the polar decomposition of the operator $Z \in \mathcal{G}$. Then $|Z|=U^{*} Z$. To check (iv), we first observe that $\rho\left(Z^{*}\right)=\rho(Z)$. By Lemma 3.1 we have

$$
\begin{aligned}
\underline{\rho(|Z|)} & =\inf \left\{\rho_{1}(X)+\rho_{2}(Y): X \in \mathcal{E}, Y \in \mathcal{F} \text { and }|Z|=X+Y\right\} \\
& \geqslant \inf \left\{\rho_{1}(X)+\rho_{2}(Y): X \in \mathcal{E}, Y \in \mathcal{F} \text { and } U|Z|=U X+U Y\right\} \\
& \geqslant \inf \left\{\rho_{1}(U X)+\rho_{2}(U Y): X \in \mathcal{E}, Y \in \mathcal{F} \text { and } U|Z|=U X+U Y\right\} \geqslant
\end{aligned}
$$




$$
\begin{aligned}
& \geqslant \rho(Z)=\inf \left\{\rho_{1}(X)+\rho_{2}(Y): X \in \mathcal{E}, Y \in \mathcal{F} \text { and } Z=X+Y\right\} \\
& \geqslant \inf \left\{\rho_{1}(X)+\rho_{2}(Y): X \in \mathcal{E}, Y \in \mathcal{F} \text { and }|Z|=U^{*} Z=U^{*} X+U^{*} Y\right\} \\
& \geqslant \inf \left\{\rho_{1}\left(U^{*} X\right)+\rho_{2}\left(U^{*} Y\right): X \in \mathcal{E}, Y \in \mathcal{F} \text { and }|Z|=U^{*} X+U^{*} Y\right\} \\
& \geqslant \inf \left\{\rho_{1}(T)+\rho_{2}(S): T \in \mathcal{E}, S \in \mathcal{F} \text { and }|Z|=T+S\right\}=\rho(|Z|)
\end{aligned}
$$

and this proves (iv).

In order to check (i), we choose $A, B \in \mathcal{E}^{+}, A \leqslant B$. By Lemma 2.1, the identity $A=V B V^{*}$ holds for some $V \in \mathcal{M}_{1}$. Let $\alpha=\rho(B)$. Then for each $\varepsilon>0$ there exist operators $X_{\varepsilon} \in \mathcal{E}$ and $Y_{\varepsilon} \in \mathcal{F}$ such that

$$
B=X_{\varepsilon}+Y_{\varepsilon}, \alpha \leqslant \rho_{1}\left(X_{\varepsilon}\right)+\rho_{2}\left(Y_{\varepsilon}\right)<\alpha+\varepsilon .
$$

Then $A=V\left(X_{\varepsilon}+Y_{\varepsilon}\right) V^{*}$ and by Lemma 3.1 we have

$$
\rho(A) \leqslant \rho_{1}\left(V X_{\varepsilon} V^{*}\right)+\rho_{2}\left(V Y_{\varepsilon} V^{*}\right) \leqslant \rho_{1}\left(X_{\varepsilon}\right)+\rho_{2}\left(Y_{\varepsilon}\right)
$$

By an arbitrary choice of the number $\varepsilon>0$ this completes the proof.

Proposition 4.1. Let $\mathcal{E}, \mathcal{F}$ be ideal spaces on $(\mathcal{M}, \tau)$ and $\mathcal{G}=\mathcal{E}+\mathcal{F}$. If the mappings $\rho_{1}: \mathcal{E} \rightarrow[0,+\infty]$ and $\rho_{2}: \mathcal{F} \rightarrow[0,+\infty]$ satisfy condition (v) (respectively, (vi)), then the mapping $\rho: \mathcal{G} \rightarrow[0,+\infty]$ defined by formula (2) also satisfies condition (v) (respectively, (vi)).

Example 4.1. The identity $\mathcal{E}+\mathcal{F}=\widetilde{\mathcal{M}}$ holds for $\mathcal{E}=\mathcal{M}$ and $\mathcal{F}=\widetilde{\mathcal{M}}_{0}$ [16]. The topology $t_{\tau}$ can be defined by means of an ideal F-norm

$$
\rho_{\tau}(X)=\inf _{t>0} \max \left\{t, \mu_{t}(X)\right\}, \quad X \in \widetilde{\mathcal{M}} .
$$

For $Z \in \widetilde{\mathcal{M}}$ we define (cf. formula (2))

$$
\rho(Z)=\inf \left\{\|X\|+\rho_{\tau}(Y): X \in \mathcal{M}, Y \in \widetilde{\mathcal{M}}_{0} \text { and } Z=X+Y\right\} .
$$

Then $\rho$ satisfies conditions (iv) and (v). The restriction $\left.\rho\right|_{\widetilde{\mathcal{M}}^{+}}$satisfies conditions (i)-(iii). Thus, the mapping $\rho: \widetilde{\mathcal{M}} \rightarrow \mathbb{R}^{+}$is an ideal F-norm majorizing $\rho_{\tau}$. Since

$$
\|X\|=\lim _{t \rightarrow 0+} \mu_{t}(X)=\sup _{t>0} \mu_{t}(X) \geqslant \rho_{\tau}(X) \quad \text { for each } \quad X \in \mathcal{M},
$$

we have $\rho(Z) \leqslant 2 \rho_{\tau}(Z)$ for each $Z \in \widetilde{\mathcal{M}}$.

Remark 4.1. Let $\mathcal{E}, \mathcal{F}$ be ideal spaces on0 $(\mathcal{M}, \tau)$ and $\mathcal{G}=\mathcal{E} \cap \mathcal{F}$. If mappings $\rho_{1}: \mathcal{E} \rightarrow$ $[0,+\infty]$ and $\rho_{2}: \mathcal{F} \rightarrow[0,+\infty]$ satisfy one of conditions (i)-(vi), then the mapping $\rho: \mathcal{G} \rightarrow$ $[0,+\infty]$ defined by the formula $\rho(Z)=\max \left\{\rho_{1}(Z), \rho_{2}(Z)\right\}$ for each $Z \in \mathcal{G}$ satisfies this condition as well.

Acknowledgments The author thanks Professor V.I. Chilin for valuable advices.

\section{BIBLIOGRAPHY}

1. I.E. Segal A non-commutative extension of abstract integration // Ann. Math. 57:3, 401-457 (1953).

2. A.M. Bikchentaev. Ideal spaces of measurable operators affiliated to a semifinite von Neumann algebra // Sibir. Matem. Zhurn. 59:2, 309-320 (2018). [Siberian Math. J. 59:2, 243-251 (2018).]

3. A.F. Ber, G.B. Levitina, V.I. Chilin. Derivations with values in quasi-normed bimodules of locally measurable operators // Matem. Trudy. 17:1, 3-18 (2014). [Siberian Adv. Math. 25:3, 169-178 (2015).]

4. A.M. Bikchentaev. Triangle inequality for some spaces of measurable operators // Konstrukt. Teor. Funkt. Funkt. Anal. 8, 23-32 (1992). (in Russian). 
5. A.M. Bikchentaev. On noncommutative function spaces // in "Selected Papers in $K$-theory". Amer. Math. Soc. Transl. Ser. 2. 154, 179-187 (1987).

6. M. Takesaki. Theory of operator algebras. I. Encyclopaedia of Mathematical Sciences. 124. Operator Algebras and Non-commutative Geometry 5. Springer-Verlag, Berlin (2002).

7. E. Nelson. Notes on non-commutative integration // J. Funct. Anal. 15:2, 103-116 (1974).

8. F.J. Yeadon. Non-commutative $L^{p}$-spaces // Math. Proc. Cambridge Phil. Soc. 77:1, 91-102 (1975).

9. A.M. Bikchentaev. On a property of $L_{p}$ spaces on semifinite von Neumann algebras // Matem. Zamet. 64:2, 185-190 (1998). [Math. Notes, 64:2, 159-163 (1998).]

10. I.C. Gohberg, M.G. Krein. Introduction to the theory of linear nonselfadjoint operators. Nauka, Moscow (1965). [Transl. Math. Monogr. 18. Amer. Math. Soc. Providence, RI (1969).]

11. F.J. Yeadon. Convergence of measurable operators // Proc. Cambridge Phil. Soc. 74:2, 257-268 (1973).

12. P.G. Dodds, T.K.-Y. Dodds, B. de Pagter. Noncommutative Köthe duality // Trans. Amer. Math. Soc. 339:2, 717-750 (1993).

13. C.A. Akemann, J. Anderson, G.K. Pedersen. Triangle inequalities in operator algebras // Linear Multilinear Algebra. 11:2, 167-178 (1982).

14. V.I. Chilin. Triangle inequality in algebra of locally measurable operators // in "Matematicheskii analiz i algebra". Collection of scientific works of Tashkent Univ. Tashkent Univ. Publ., Tashkent, 77-81 (1986). (in Russian).

15. A.M. Bikchentaev. Minimality of convergence in measure topologies on finite von Neumann algebras // Matem. Zamet. 75:3, 342-349 (2004). [Math. Notes. 75:3, 315-321 (2004).]

16. A. Stroh, Grame P. West. $\tau$-compact operators affiliated to a semifinite von Neumann algebra // Proc. Roy. Irish Acad. Sect. A. 93:1, 73-86 (1993).

Airat Midkhatovich Bikchentaev,

Kazan Federal University,

Kremlevskaya str. 18,

420008, Kazan, Russia

E-mail: Airat.Bikchentaev@kpfu.ru 